\title{
Imaging in Radiation Oncology: Time to Exercise Constrain
}

\author{
Suhag $\mathrm{V}^{1^{*}}$, Sunita BS ${ }^{2}$, Singh $\mathrm{AK}^{3}$, Sunita Dashottar ${ }^{4}$ and Maj Nishant Lohia ${ }^{5}$ \\ ${ }^{1}$ HOD Radiotherapy, Army hospital R \& R, Delhi, India \\ ${ }^{2}$ Professor of Pathology, Base Hospital, Delhi, India \\ ${ }^{3}$ Senior advisor Radiodiagnosis and Imaging, Army hospital $R \&$ R, Delhi, India \\ ${ }^{4}$ Classified Specialist Radiodiagnosis and Imaging, Military Hospital CTC Pune, India \\ ${ }^{5}$ Resident Radiotherapy, Army hospital R \& R, Delhi, India
}

"Corresponding author: Virender Suhag, HOD Radiotherapy, Army Hospital (R\&R), Dhaula Kuan, Delhi Cantt, Delhi, India, Tel: +918826804584; E-mail: virendersuhag@gmail.com

Received date: May 18, 2015, Accepted date: May 19, 2015, Publication date: May 25, 2015

Copyright: (c) 2015 Suhag V, et al. This is an open-access article distributed under the terms of the Creative Commons Attribution License, which permits unrestricted use, distribution, and reproduction in any medium, provided the original author and source are credited.

\section{Opinion}

The field of Radiation Oncology has witnessed a sea change over the past two decades with the advent of newer techniques to practice highest form of conformal radiotherapy (RT). There has been a dramatic shift from conventional 2-dimensional RT on Telecobalt machines to 3-dimensional and 4-dimensional RT on Linear accelerators. The advancement of medical imaging has been critical in helping to achieve this change. Radiotherapy, more then any other treatment modality, relies heavily and often exclusively on medical imaging to determine the extent of disease and the spatial relation between target region and neighbouring healthy tissues. The invention of computed tomography (CT) was pivotal in the development of treatment planning. Despite some disadvantages, CT remains the only three-dimensional imaging modality used for dose calculation. Newer image modalities, such as magnetic resonance (MR) imaging and positron emission tomography (PET), are also used secondarily in the treatment-planning process. PET also provides metabolic information to supplement the CT and MR anatomical information. Currently, radiation therapy linacs are fully controlled by computers and with new techniques of dose delivery, such as intensity-modulated radiation therapy (IMRT), volumetric-modulated arc therapy (VMAT), and imaged-guided radiotherapy (IGRT), the treatment delivery precision is measured in millimeters. The upcoming concept of multimodality scanners such as PET/CT and PET/MR will further revolutionalize optimal tumor targeting information.

The imaging modalities are vital for the initial diagnostic workup of the primary, nodal and metastatic disease; to plan and execute the newer technologies of RT like IMRT, IGRT, stereotactic radiosurgery (SRS), Gamma knife/cyber knife based treatment, stereotactic body radiotherapy (SBRT) etc; to conduct guided aspirations and biopsies and intervention-based palliative procedures; and also to differentiate post-RT changes and necrosis from viable residual tumor or recurrence by magnetic resonance angiography and spectroscopy (MRA, MRS), SPECT etc. There is no denying the fact that these imaging modalities are vital to ensure precision in radiotherapy and to provide good quality of life to the patients. However, there must not be repetition of imaging investigations during diagnostic workup and during surveillance. At times the over-enthusiastic Oncologist will ask for a battery of imaging tests without exercising caution and diligence; some of these investigations hardly contribute any information towards management of the case.

However, there are certain problems and challenges being faced in practicing purely image based Radiotherapy in developing countries which need to be addressed. The first and the foremost is more and more dependence of the Radiation Oncologists on the imaging modalities during workup, planning and follow-up of oncology cases; which is gradually replacing the clinical acumen and skills. One should not forget that there is no substitute to clinical examination and individualization of treatment protocol. The oncologists need to spend more time with the patients and their relatives, making best use of their soft skills and the clinical knowledge. Referring the patient from one imaging department to other may lead to fatigue and irritation in the distressed cancer patient.

The second challenge being faced is that too many requisitions for imaging are over burdening our diagnostic colleagues. In high volume centers, the specialists from imaging department have to work more than 10 hours a day and report over 30 scans. It may hinder preventive maintenance and quality assurance, and increases the likelihood of reporting errors; apart from causing physical and mental stress in the reporting staff. Another area of concern is long waiting period for imaging appointment, especially in public sector, at times spanning over 2-4 weeks. It may increase the risk of disease progression if the workup and RT planning is delayed beyond a reasonable waiting time.

In most developing countries including India, there is hardly any exposure of the residents how to interpret and report the newer modalities of imaging. On an average, the oncology residents undergo their rotation in radio-diagnosis and nuclear medicine department for 3-4 weeks. There is no structured teaching program for these residents as most of the consultants and senior faculty of these departments are busy reporting their images. It is recommended that the authorized governing bodies, like Medical Council of India and National Board of Examinations, must ensure that the oncology residents actually undergo a vigorous 3-month training capsule to learn various imaging modalities. The Medical physicists, dosimetrists and RT technologists should also undergo periodic rotations in these departments to refresh and update their existing skills.

Too frequent and too many imaging modalities substantially increase the cost of medical care. In most developing countries, most of the patients are not insured under any public or private insurance scheme and shell out hefty amounts out of their pockets. The socioeconomic condition of the patient's families should be kept in mind while initiating demand for any imaging. At times, ordering too many investigations may lead to trust deficit between the patient and doctor and may lead to a conflict. Moreover, there is a great diversity in the availability of the novel imaging and treatment techniques in the private and public sectors of most developing countries. It is high time 
Citation: Suhag V, Sunita BS, Singh AK, Dashottar S, Lohia MN (2015) Imaging in Radiation Oncology: Time to Exercise Constrain. J Nucl Med Radiat Ther 6: 228. doi:10.4172/2155-9619.1000228

Page 2 of 2

the respective governments take the plunge to ensure uniform standard-of-care for the rich and poor alike. More imaging centers need to be opened in suburban and rural areas; and also in the government hospitals and medical colleges. The government should also ensure that there is no malpractice while asking for these imagebased investigations.

To sum up, radiation oncology is more dependent on medical imaging than it has ever been, and that dependence is only going to become greater. The Oncology fraternity should come together and formulate their own institutional guidelines regarding imaging and should be more judicious and practical while asking for these imaging investigations. This issue must be exhaustively debated in the upcoming seminars and conferences. 